\title{
L'HOMME DE DIDEROT
}

En quoi Diderot était-il donc un philosophe? On se représente souvent le philosophe comme un sage vieillard, ou un homme vieux avant l'âge, enfin assagi, plongé dans une méditation solitaire, à l'écart du commun des mortels, patient et doux, inutile et inoffensif, poursuivant ses spéculations ou ses ruminations intellectuelles dans le silence et l'obscurité, ou plutôt dans la semi-obscurité, dans ce clair-obscur où Rembrandt, songeant sans doute à Spinoza, a préféré le peindre. Une sorte de moine laïque, ou plutôt, puisque la solitude lui est si précieuse, une sorte d'ermite de la pensée rationnelle, revenu de tout pour n'aller décidément qu'en lui-méme. Et nous voyons bien en cela l' image d'une philosophie capable de présenter ses titres de gloire.

Mais, Descartes l'avait déjà remarqué :

Au lieu de celle philosophic spéculative qu'on enseigne dans les écoles, on en peut trouver une pratique, par laquelle, connaissant la force et les actions du feu, de l'eau, de l'air, des astres, des cieux et de tous les autres corps qui nous environnent, aussi distinctement que nous connaissons les divers métiers de nos artisans, nous les pourrions employer en méme façon à tous les usages auxquels ils sont propres, et ainsi nous rendre comme maîtres et possesseurs de la nature".'

Eh bien! Diderot élabore exemplairement une telle philosophie pratique. Avec tous ceux que, dans la seconde moitié du XVIIIe siècle en France, on a appelé les "philosophes", nous avons affaire à des hommes qui ne se soucient guère de ressembler aux méditatifs à la Rembrandt, mais qui vivent, et qui vivent dans leur temps, qui en épousent les querelles, qui interviennent sans cesse dans le monde, par la pensée et par l' expression de la penséc, par la parole et par l'écrit. Ils préparent un monde nouveau; le monde nouveau qui va bientôt surgir, et qui les aurait sans doute beaucoup surpris cependant, et beaucoup déçus, s'ils avaient pu assister à sa naissance : Voltaire, Rousseau, Helvétius, et d'autres...

Plus tard, Gavroche chantera : 134.

${ }^{1}$ Rene Descartes. Discours de la methode, in Oeuvres, Coll. Pléiade, Paris, 1937, p. 
"Je suis tombé par terre

C'est la faute à Voltaire

Le nez dans le ruisseau

C'est la faute à Rousseau!... ${ }^{2}$

Pourquoi donc Victor Hugo, en 1862, n'a-t-i 1 pas mis plutôt, sur les lèvres du jeune héros mourant, le nom de Diderot ? Pourquoi Diderot n'a-t-il pas compté immédiatement, du moins au même degré, dans l' imagination populaire et dans l'histoire savante, parmi ceux qui ont formé la pensée de leur temps, parmi ceux qui ont infléchi l'histoire ?

On ne l'ignorait, ni ne le méprisait certes pas !

Mais, de toute évidence, en ce qui concerne le génie, l'oeuvre et l' influence, on le plaçait derrière Voltaire ou Rousseau. Or, voici que se produit un reclassement des valeurs, dont témoigne l'immensité de la commémoration du bi-centenaire de la mort de Diderot. Pour nous, maintenant, c'est Diderot qui est le plus grand. Il s'instaure même une sorte de culte de Diderot - ce qui, touchant ce mécréant, ne manque pas de piquant.

Chaque époque se souvient des hommes qu'elle choisit dans le passé, selon son goût et son humeur. Nous ne commémorons que ceux que nous aimons; ceux dont nous croyons qu'ils ont préparé et produit ce que nous sommes; ceux en qui, à quelques égards, nous pouvons nous reconnaître. Nous ne commémorons que nous-mêmes. Comment pouvons -nous nous reconnaître en Diderot, et en son oeuvre ? Quel était donc l'homme de Diderot, auquel nous ressemblerions comme des frères?

Chronologiquement, Diderot est le philosophe des Lumières, le plus typique. Ce grand mouvement intellectuel de libération de l'esprit, de lutte contre les oppressions religieuses et politiques, d' exaltation de l' esprit critique, de la raison, de la science, que l'on appelle les Lumières, on le situe communément, en France, entre 1715 (la mort de Louis XIV et la fin du Grand Siècle - or Diderot est né en 1713) et 1789 (la Révolu-tion française, tenue, non sans quelques illusions, pour le triomphe des Lumières). Or Diderot est mort en 1784. Les Lumières et Diderot ont vieilli ensemble.

Dans ce courant intellectual, social, antireligieux, politique, philosophique que sont les Lumieres, Diderot va le plus loin, se montre le plus audacieux. En philosophie spéculative,qu'il pratique aussi, il avance jusqu' au matérialisme, mais un matérialisme chaleureux, bien différent de ce que l'on a pu appeler "le matérialisme triste" de

\footnotetext{
2 Victor Hugo, dans Les Miserables.
} 
d'Holbach. Un matérialisme sensible, imaginatif, poétique, vitaliste, débordant largement le mécanisme passif et statique du matérialisme traditionnel - matérialisme qui ne réussit à s'exprimer convenablement que dans des dialogues animés et passionnés, tels que Le Rêve de d'Alembert.

Mais déjà il nous faut noter que Le Rêve de d'Alembert, mis récemment en scène à Paris, ne fut publié qu'en 1830, près de cinquante ans après la mort de son auteur !

Toutefois, même dans des oeuvres moins attachantes que $L e$ Rêve de d'Alembert, Diderot s'offrait bien à ses contemporains comme l'écrivain critique, irrespectueux et même scandaleux. Aussi fut-il, pour le punir de sa Lettre surr les avengles, emprisonné à Vincennes où Rousseau vint lui faire une visite riche de conséquences. A ce titre de défenseur de la liberté spirituelle, de la liberté de conscience et d'expression, de ce que nous appelons maintenant les "droits de l'homme", il a mérité la haine des pouvoirs du temps, de leurs représentants officiels, de leurs valets de plume, alors plus influents et plus célèbres que lui, bien oubliés depuis. Qui se souvient de Palissot, si ce n'est parce que Diderot l'a combattu ?

Jamais les adversaires de Diderot ne désarmèrent. Au long des siècles, ils l'attaquent violemment, infatigablement, fanatiquement. Ainsi, en 1880, quelques années avant le centième anniversaire de la mort de Diderot, qui ne fut ccmmémoré que très discrètement et précairement, Barbey d'Aurevilly dénonçait encore le grand méchant homme, et en quels termes!

Impossible, se plaignait-il, d'écrire l'histoire du XVIIle siecle sans y
rencontrer le nom et l' influence de Diderot, de l'infatigable ouvrier
de l'Encyclopédie qui a entassé la vidange de toutes les erreurs du
XVIIIe siècle dans cette infecte tine de l'Encyclopédie, mais non pas
pour les emporter ! en cela inférieur aux autres ouvriers de ces
dégoutantes besognes... Diderot, surfait, grandi pour mille raisons dont
la plus forte et la plus actuelle est ce matérialisme qui produit des
Littre en philosophie, des Courbet en art et des Zola en littérature,
déjections dernieres !

Et en 1913, alors que certains suggéraient de fêter nationalement le 2è centenaire de la naissance de Diderot, Maurice Barrès, souvent mieux inspiré, c'est bien le cas de le dire, s'indignait :

Vraiment, c'est une idée malheureuse qu'avait le Sénat, de vouloir

${ }^{3}$ J. Barbey d'Aurevilly, Goethe et Diderot. Paris: 1880, p. 141 et 147. 
célébrer une fête nationale du deuxième centenaire de Diderot... L'apothéose de Rousseau fut une erreur... Pourquoi, diable ! allez-vous chercher dans le vieil arsenal de nos guerres civiles des hommes drapeaux, des revenants incapables de susciter et de diriger nos forces d'enthousiasme ?" 4

Et, par opposition, Barrès trouve sa satisfaction dans le fait que "le gouvernement de la République a rendu un éclatant hommage au génie de Mistral appuyé sur l'une de nos plus belles provinces"s.

En 1913, Barrès place le petit Mistral - bien sympathique au demeurant - au dessus de l'immense Diderot. Il se produit parfois des ratés dans le fonctionnement des meilleurs esprits.

L'homme auquel s'attachent des haines aussi vives et tenaces, présentait pourtant un visage capable de susciter toutes les sympathies.

Un homme étrange, ce Diderot, un peu déconcertant, mais, aussi, fascinant. Voici comment Joseph Garat raconte sa première rencontre avec Diderot.

Garat, alors fort jeune, n'était pas encore entré dans la tragédie de l'histoire. C'est lui qui, plus tard, conventionnel régicide et ministre de la justice du gouvernement révolutionnaire, sera chargé d'annoncer à Louis XVI sa condamnation à mort, et que les révolutionnaires ont décidé de guillotiner la monarchic en sa personne. Scène où se drape la sublimité de l'histoire.

Mais quand, encore jeune, il se présente à Diderot, c'est en toute modestie et en toute simplicité, et il donne de cette rencontre un récit spontané, il trace un portrait pris sur le vif.

Diderot et Garat ne s'étaient encore jamais vus. Diderot ne demande à Garat, qui vient d'entrer dans la piece, ni son nom ni l'obiet de sa visite. Et voici ce qui nous serait arrivé, si nous nous étions présentés nous-mêmes à Diderot, comme le fit ce jeune inconnu, Garat:

Il devine et m'épargne la peine de balbutier tout cela...; il se lève et ses yeux se fixent sur moi, et il est très clair qu'il ne me voit pas du tout.Il commence à parler, mais d'abord si bas et si vite, que, quoique je sois près de lui, quoique je le touche, j'ai peine à l' entendre et à le suivre... Peu à peu sa voix s'élève et devient distincte et sonore ; il était d'abord immobile, ses gestes deviennent frequents et animés; il ne m'a jamais vu que dans ce moment, et lorsque nous sommes debout, il m'environne de ses bras ; quand nous sommes assis, il

\footnotetext{
${ }^{4}$ L'Oeuvre de Maurice Barrès, tome XII. Paris: Club de l'Honnête Homme, 1967, p. 97 et p. 150.

${ }^{5}$ Ibid., p. 100.
} 
frappe sur ma cuisse comme si elle était à lui. Si les liaisons legères et rapides de sa conversation amènent le mot de lois, il me fait un plan de législation; si elles amènent le mot drame, il me donne à choisir entre cinq ou six plans de drames ou de tragedies. A propos de tableaux qu'il est necessaire de mettre sur le théatre, il se rappelle que Tacite est le grand peintre de l'antiquité, et il me récite ou me traduit les Annales et les Histoires..

\section{A propos de Tacite :}

Plusieurs chefs-d'oeuvre de ce grand homme sont perdus, "mais on pourra les retrouver" et cette espérance le transporte de joie... Il me joue une scène entière de Térence ; il chante presque plusieurs chansons d'Horace... Beaucoup de monde entre alors dans son appartement, et le bruit des chaises le fait sortir de son enthousiasme et de son monologue ; il me distingue au milieu de la compagnie, et vient à moi comme à quelqu'un que l'on retrouve après l'avoir vu autrefois avec plaisir; il a connu qu'il y avait beaucoup à gagner dans ma conversation... en me quittant, il me donne deux baisers sur le front, et arrache sa main de la mienne avec une douleur veritable"...6

Ce portrait confirme assez bien l' estimation que Diderot faisait de lui-même.

Il était fier d'être né à Langres, et il décrivait ainsi les Langrois: "La tête d'un Langrois est sur ses épaules comme un coq d'église au haut d'un clocher : elle n'est jamais fixe dans un point ; et si elle revient à celui qu'elle a quitté, ce n'est pas pour s'y arrêter".

Et il ajoute : "Pour moi, je suis de mon pays"7.

De tels portraits et de tels aveux poussent à la caricature certains traits de Diderot : un homme-girouette, instable, impulsif, incapable d'approfondir une idée et de s'y arrêter, et surtout incapable de ne pas dire aussitôt ce qui lui passe par la tête, frivole et indiscret, léger et expansif. Et certes on retrouve beaucoup de cela dans son oeuvre, et peut-être principalement dans le personnage du neveu de Rameau.

Encore convient-il de remarquer que cette girouette ne tournait pas à n'importe quel vent ! I1 y a des girouettes qui ne savent rien de Tacite ou de Térence, qui ne composent ni drames, ni poèmes... Diderot avait été, à Langres, le meilleur élève des Jésuites.

Une girouette quelque peu plébéienne, un naturel impertinent,

- Cité dans l'article "Garat": Biographie universelle, Ancienne et moderne, de Michaud. Paris: Deplace, 1856, tome XV, p. 527.

${ }^{7}$ Cité par G. Lanson. Histoire de la litterature française, remaniee par P. Tuffrau. Paris: Hachette, 1952, p. 740. 
- aisément familier, un peu vulgaire.

Même Catherine II, la Grande Catherine, l'Impératrice de toutes les Russies, qui admirait Diderot, et avait voulu en orner sa cour, pendant un temps, s'offusquait, comme Garat, de la manière dont Diderot lui tapait sur les cuisses, au cours de leurs conversations philosophiques amicales. Et pourtant les cuisses de Catherine, si l'on ose dire, en avaient vu bien d'autres...

Mais venant d'un philosophe, du directeur de l'Encyclopédie, certains gestes, certaines paroles, certains opuscules, choquaient - et ceci même dans un siècle où la fiction des Bijoux indiscrets ne pouvait en aucune manière dépasser, dans la pornographie ou la pornophonie, la réalité des pratiques de la Régence ou du règne du Bien-Aimé...

A la lecture de ces témoignages sur la mobilité d' esprit de Diderot, impulsif et variable, primesautier, volontiers polisson, on ne peut s'empêcher de penser qu'il y a un mystère Diderot. Qui était donc Diderot, et quelle était donc sa vision propre de l'homme, et de l'homme dans le monde?

Car, tout de même, cette girouette, ce polisson, Diderot, c'est lui qui sut mener à bonne fin la gigantesque entreprise de l'Encyclopedie, au cours de longues années d'application, de travail, cet énorme Dictionnaire raisonné des sciences, des arts et des métiers, qu'il a porté sur ses épaules comme, dit un de ses biographes, le géant Atlas portait sur ses épaules le monde.

Peut-on imaginer oeuvre plus massive, plus sérieuse, plus austère que l'Encyclopédie?? On n'y éclate pas de rire à chaque détour de page!

L'élaboration, la création de l'Encyclopédie exigea un labeur immense - du simple point de vue administratif, technique, financier, rédactionnel - et l'on peut à peine imaginer ce que cet homme, à lui seul, a su accomplir à cet égard.

Grand livre de science, de technique et de pratique, mais aussi machine de guerre de la secte des philosophes pour la libération intellectuelle, contre la religion, les superstitions, le despotisme, les privilèges, les abus, en faveur de la vérité et de la justice - l'Encyclopédie se heurta à la résistance acharnée des pouvoirs établis. Diderot déclarait que "le premier pas vers la philosophie c'est l'incrédulité"8 "qu'il faut tout examiner sans exception et sans ménagement... il faut renverser les barrières que la raison n' aura pas posées"...9

${ }^{8}$ Dictionnaire des philosophes, article "Diderot". Paris: P.U.F., 1984. p. 739.

${ }^{9}$ Cité par Lanson-Tuffrau, op. cit., p. 423. 
Au procès qui fut instruit contre l'Encyclopédie et ses auteurs, l'avocat général dénonçait, à juste titre "une société formée pour soutenir le matérialisme, pour détruire la religion, pour inspirer l'indépendance" ... ${ }^{10}$

L'Encyclopédie fut certes soutenue et aidée par quelques personnages influents, d'Argenson notamment, et la Pompadour, qui avait autant d' esprit que de beaute. Faibles protections. Diderot dut lutter pour l'Encyclopédie, avec courage, constance, obstination, dévouement total à la cause qu'il avait choisie. La girouette ne tournait plus, même dans la tempête.

Diderot a lui-même résumé et raconté cette passion, et tous les témoignages et documents connus confirment les jugements qu'il porte, sur cette affaire, dans un Mémoire pour Catherine II :

\begin{abstract}
J'ai travaille près de trente ans à cet ouvrage. De toutes les persécutions qu'on peut imaginer, il n'en est aucune que je n'aie essuyee. Je laisse là les libelles diffamatoires de toutes couleurs. J'ai été exposé à la perte de l'honneur, de la fortune et de la liberté. Mes manuscrits circulaient de dépôt en dépôt, recélés tantôt dans un lieu, tantôt dans un autre. On a tenté plus d'une fois de les enlever. J'ai passé plusieurs nuits à ma fenêtre dans l'attente de l'exécution d'un ordre violent. J'ai été sur le point de m'expatrier, et c'était le conseil de mes amis, qui ne voyaient plus de sarete à Paris pour moi. L'ouvrage a été proscrit et ma personne menacée par différents édits du roi et par plusieurs arrêts du Parlement. Nous avons eu pour ennemis déclarés la cour, les grands, les militaires, qui n'ont jamais d'autre avis que celui de la cour, les prêtres, la police, les magistrats, ceux d'entre les gens de lettres qui ne cooperaient pas à l'entreprise, les gens du monde, ceux d'entre les citoyens qui s'étaient laissé entraîner par la multitude. Cependant, au milieu de ce déchaînement général, tout le monde souscrivait. Ils voulaient avoir l'ouvrage et perdre les auteurs. Lorsqu'on eut inutilement employe tous les moyens d'empêcher l'ouvrage, on ne songea plus qu'à ralentir son execution et à nuire à sa perfection. Nous avons souffert des suspensions de plusieurs années et des désertions de cooperateurs, et, pour comble de disgrâce, un infâme imprimeur, qui dépeçait mon ouvrage à mon insu pendant la nuit, a mutile dix volumes et brolé les manuscrits qu'il ne jugeait pas à propos d' employer.

On fit du nom d'encyclopédiste une étiquette odieuse (...). Il restait une dernière ressource, c'etait de nous rendre ridicules..."11
\end{abstract}

${ }^{10}$ Cité par Lanson et Tuffrau.

${ }^{11}$ Diderot. Mémoires pour Catherine de Russie, in Oeuvres. Paris: Le Club du Livre Française, tome X, 1971, pp. 791-792. 
Ainsi Diderot dresse-t-il le répertoire de ses ennemis, en tombant du plus haut, le roi et la cour, au plus bas, les comédiens et Palissot !!

Et pour attester de la constance de Diderot, de la fermeté de ses idées et de ses intentions, n'oublions pas qu'il a toute sa vie durant travaillé au projet d'un grand Traité de morale. S'il n'a pas réussi à l'écrire finalement, ce n'est pas par paresse ou versatilité - mais évidemment pour des raisons profondes.

Diderot était un esprit vivant, contradictoire, multiple et riche semblable à l'homme qu'il décrit dans quelques unes de ses oeuvres.

Et c'est ici que nous rencontrons le deuxième mystère de Diderot, plus important et plus significatif.

Ces oeuvres, dans lesquelles Diderot a mis en scène l'homme tel qu'il le concevait, les contemporains de Diderot ne les ont pas connues. Or ce sont celles qui suscitent le plus vivement notre intérêt.

Alors que tout le monde savait presque tout, à chaque instant, des faits et gestes de Voltaire, ce clandestin accompli, personne n'a rien su, et pendant trés longtemps, de ce Diderot, que l'on tient un peu rapidement pour un bavard, un indiscret, incapable de se retenir de parler.

De ce que nous aimons et apprécions dans son oeuvre, il n'a lui-même rien publié. C'est un auteur secret. Songez-y : Jacques Le Fataliste, écrit en 1773, ne paraîtra qu'en 1793, dix ans après la mort de Diderot. La Religieuse, ne sera publiée qu'en 1796 ! Le Rêve de d'Alembert, le Paradoxe sur le comédien ne seront connus du public qu'en 1830 , près de 50 ans après la disparition de leur auteur. Le Neveu de Rameau, écrit en 1762, paraîtra d'abord en langue allemande, dans la traduction de Goethe, en 1805 . Il sera retraduit de l'allemand en français en 1821 - soixante ans après avoir été rédigé. Le manuscrit authentique ne sera retrouvé, par hasard, qu'en 1891 !

Avant sa mort, Diderot, en tant que romancier et dialoguiste, n'était connu que par les Bijoux indiscrets !

Cet homme des Lumières a préféré laisser dans l'ombre la silhouette de l'homme, tel qu'il concevait celui-ci. Il a confié à la postérité le soin de découvrir cet homme - non sans grands risques de perte, d'oubli, ou d'incompréhension. Alors, si l'homme Diderot, et si l'homme de Diderot sont restés si longtemps secrets, il doit y avoir à cela des raisons. Pourquoi Diderot n'a-t-il pas affiché et exposé l'homme tel qu'il le comprenait, alors qu'il affichait et exposait si efficacement les métiers et les choses, tels qu'il les voyait. On peut, à ce sujet, hasarder quelques hypotheses.

A cette discrétion, à ce secret de Diderot, on peut sans doute attribuer d'abord des causes positives. Diderot avait l'expérience de la 
répression, de la censure, et même de l'emprisonnement. S'il s'est abstenu de publier La Religieuse ou le Neveu de Rameau, ouvrages à certains égards scandaleux, c'est qu'il n'avait pas envie de retourner en prison. C'est par prudence, ou par peur, qu'il aurait renoncé à la publication de telles oeuvres.

Cette explication ne me semble pas plausible. La crainte a pu jouer un rôle d'appoint, dans la décision de Diderot. Mais, nous l'avons vu, il etait courageux, combattif, un lutteur. La crainte ne pouvait l'emporter, dans son esprit, sur la passion de la vérité et de la liberté. Et puis, si cela avait malgré tout été le cas, plutôt que de renoncer à publier ces grands ouvrages, il ne se serait pas donné la peine inutile de les composer et de les écrire!

Une autre raison de cette abstention, aurait été le refus de se mettre à dos ses amis - ou ses complices. Le fatalisme de Jacques, les conséquences perverses de l'éducation du Neveu, en un mot, l' orientation de la pensée de Diderot, dans ces dialogues, ou dans ces romans dialogués, allait au rebours des Lumières, et signalait en elles, un fâcheux revers d'ombre.

Il est possible que de telles considérations aient joué un rôle, elles-aussi, dans la détermination de Diderot. Mais on peut les compenser, d'un autre côté, en se souvenant que Diderot, à la fin de sa vie, avait déjà rompu avec presque tous ses premiers compagnons de lutte, qu'il n'était pas homme à renoncer à une idee pour l'amour ou le respect des personnes, et que, plus généralement, il connaissait la fecondité et la nécessité des débats, des discussions, des oppositions intellectuelles. Non, ni la crainte de la police et de la justice, ni la peur de déplaire à des amis n'ont pu, à elles seules, l'empêcher de faire éditer ses oeuvres les plus significatives et les plus belles.

Alors voici une explication possible. La raison profonde pour laquelle Diderot a gardé secrets ses manuscrits et a dissimulé la conception qu'il se faisait de l'homme, c'est que celle-ci d'abord entrait en contradiction avec beaucoup des idées qu'il avait soutenues et continuait à soutenir lui-même. S'il gardait sa conception de l'homme secrète pour les autres, c'est aussi qu'elle restait à certains égards secrète pour lui-même. De plus, l'homme tel que Diderot le présentait - en particulier dans le Neveu de Rameau, n'était déjà plus l'homme de son temps, mais comme un pressentiment, et assez terrifiant, de l'homme de l'avenir.

Dans l'Encyclopédie déjà, on peut remarquer une étonnante opposition entre la confiance généreuse dans la science et dans les métiers que proclame l'oeuvre tout entière en une sorte d'apologie 
pratique, et les vues modestes, étriquées, et comme frileuses que Diderot développe lorsqu'il réfléchit théoriquement sur cette science et sur ces métiers. L'Encyclopédie chante ccmme un grand hymne à la gloire de la raison, de l' intelligence pratique, de leur puissance et de leurs victoires, et quand Diderot rédige lui-même l'article Art, il insiste sur leurs faiblesses, leurs limites, la mince portée de leurs effets, la soumission dernière de l'homme à la nature.

Une contradiction oppose donc l'éloge et la description complaisante de l'activité humaine, dans quelques articles du Dictionnaire, et la conception contemplative et passive de l'homme que Diderot propose, lorsqu'il en fait la théorie. Une philosophie purement empiriste et sensualiste, telle que celle de Diderot, ne peut accorder sa part à l'activité. Elle voit en l'homme essentiellement un enregistreur d'impressions, tout au plus un observateur, ou bien, car Diderot est tout de même très subtil, un réflecteur des impressions qu'il a reçues. Mais elle ne s'éleve pas jusqu'à la saisie et à l' animation du sujet actif.

Comme on est loin de l'homme de Descartes, "maître et possesseur de la nature" lorsque l'on rencontre l'homme de Diderot: ... L'homme n'est que l'interprète et le ministre de la nature..! "12

C'est la nature qui dicte et qui gouverne. Elle est le maître et le possesseur de l'homme!

Mais le plus significatif est ceci : l'Encyclopédie décrit minutieusement les métiers, les arts, les techniques, et surtout les matières premières, les outils, les instruments, les machines, les produits.

Cette minutie, ce souci de l'objet, de sa forme singulière, de son usage unique et approprié, de sa finalité concrète se révèlent très clairement dans les planches de l'Encyclopédie. Les articles de l'Encyclopédie et leurs illustrations décrivent et analysent les moyens et les fins de cette production, la production des biens de consommation et de jouissance, la production de ce que l'on appellera plus tard des valeurs d' usage, et ce que Diderot désigne comme les moyens du besoin et du luxe. Concernant ces moyens et ces résultats de la production, des arts, des métiers, Diderot va aussi loin que possible dans le détail, la singularité, la diversité, la dernière information.

Si l'on perçoit bien cette minutie, cette diversité, cette actualité des choses, dans l'Encyclopédie, alors un contraste saute à nos yeux d'hommes du XXe siècle : l'imprécision, l'uniformité, l'interchangeabilité, l'intemporalité des figures humaines, des ouvriers, ou des "manouvriers".

12 Diderot, article "Art" de IEncyclopédie. 
Le fils du coutelier de Langres a vu les couteaux et les enclumes, mais il n'a pas bien regardé son père.

Dans la mesure où il s'occupe de l'homme producteur, artisan ou manouvrier, ou manufacturier, Diderot le traite abstraitement, sans considération de temps ou de lieu, sans détermination du rapport social dans lequel il est engagé, un être abstrait, et à dire vrai, l'être abstrait qu'il lui faut pour pouvoir lui reconnaître une liberté indéfinie, universelle, elle-même abstraite.

Cette abstraction, ce goût de l' abstraction se retrouve jusque dans $L a$ Religieuse, qui raconte les malheurs d'une personne si concrète, à certains égards. Et pourtant, à y regarder de plus près, on s'aperçoit que, à la manière de bien des romanciers antérieurs, Diderot s'efforce de la dépersonnaliser. Il procède radicalement en faisant d'elle un enfant naturel. A cette époque un enfant naturel est ccmme dépourvu même de nom. Elle n'a ni nom, ni famille, ni fortune, ni métier : un être réduit romanesquementà sa plus simple expression, à son essence abstraite. On ne l'a peut-être pas assez remarqué, La Reliqieuse de Diderot, à la difference de la Marianne de Marivaux, n'a même pas à lutter pour des amours que l'on voudrait empêcher en l'enfermant au couvent. Elle n'a pas d' amours non plus. Elle n'est rien et n'a rien. Et elle combat pour la reconnaissance de sa liberté, de sa liberté abstraite, une liberté de devenir n'importe quoi parce qu'elle est plutôt une fatalité de rester rien du tout.

Dans l'Encyclopédie, c'est un être abstrait de ce genre qui réclame la satisfaction positive des besoins d'une nature humaine invariable et indifferenciée, anhistorique, et véritablement utopique. Et ceci dans une perspective purement utilitariste, ou "utiliste". L'homme abstrait soumis aux besoins qu'une nature invariable lui impose : c'est là cet "utilisme", cette pratique sordide que dénoncera plus tard Feuerbach, et sur lequel, plus tard encore, Marx rêvera de faire prévaloir une praxis sublime, la libre affirmation de soi de l'individualité humaine.

Bien sûr, Diderot ne se lance pas dans cette critique de l' abstraction et de l'utilitarisme. On peut même admettre que, dans l'Encyclopédie, il les ignore presque entièrement.

Mais voici la surprise !

Entièrement ignorés dans l'Encyclopédie, les hommes surgissent dramatiquement dans les écrits non publiés, et en particulier dans le Neveu de Rameau.

On peut dire que si Diderot, dans l'Encyclopédie, décrit le travail, le travail tel qu'il est en lui-même, producteur de biens de consommation ou de valeurs d' usage, créateurs de choses, en quelque sorte poétique, 
au sens où Aristote entend ce terme, eh bien! dans le Neveu de Rameau il envisage les effets de cette production et de cette création dans le monde humain, quand les valeurs d' usage deviennent des valeurs d'échange, quand les produits deviennent des marchandises, quand l'or devient la principale des marchandises. Alors le produit se rend maître du producteur et le neveu de Rameau peut s'exclamer : "l'or est tout!"13 L'or, le Dieu des temps modernes!

Le neveu de Rameau est un gredin, mais un gredin lucide, cynique. On peut se demander pourquoi Diderot a ainsi choisi un marginal, un parasite, comme témoin, dénonciateur et juge de l'homme moderne et de sa société. C'est parce qu'il se place à un point de vue moral, - comme le feront après lui Kant, Fichte, Hegel, - et qu'il veut condamner cette société du point de vue moral. Le Neveu de Rameau est un voleur, mais il sait qu'il vole des voleurs. C'est un parasite marginal, mais il parasite des parasites institutionnels. "Je ne m'avilis point, dit-il, en faisant comme tout le monde"14! Il se prostitue, il pousse des filles à se prostituer, mais il révère une prostitution beaucoup plus profonde, la prostitution universelle dans laquelle il lui faut bien vivre : tout est à vendre ! Les choses, bien sûr, mais aussi les oeuvres, les femmes, les hommes, les consciences, les sciences, les métiers, les arts, et les beaux arts, et bien sûr, à bas prix, Palissot...

Diderot n'a pas ignoré que lorsque l'homme change l'objet, il se change lui-même dans cette opération, et de manière imprévue et perverse - certainement pas lumineuse. Il n'a pas ignoré le déchirement dans lequel vivent les hommes parce que leur monde lui-même est déchiré - ce monde qu'ils ont créé et qu'ils se sont donné pour leur usage, ce monde de culture qu'ils opposent à la nature, et qui se retourne cependant contre eux, avec une fatalité plus pesante et plus cruelle que la nécessité naturelle.

Voila à quoi il voit aboutir l'amélioration du monde et l'éducation du genre humain que les Lumieres prétendent accompagner, ou même provoquer.

Le neveu de Rameau est un gredin, comme les autres, mais il le sait, alors que les autres gardent bonne conscience. Le neveu de Rameau est un gredin qui n'a pas réussi - et l'on s'en étonne, devant son intelligence aiguë et sa totale absence de scrupule - mais il sait qu'il est un gredin, et se tient pour tel, et donc il sait aussi qu'il y a des honnêtes

${ }^{13}$ Diderot. Oeuvres. Paris: Gallimard, Coll. Pléiade, 1951, p. 491. ${ }^{14}$ Ibid., p. 449. 
gens, qu'il existe une conscience morale, qu'il peut être l'objet d'un jugement. Il se juge. Lui-même est une conscience divisée, déchirée, sordide et à la fois impitoyable pour elle-même.

Diderot critique profondément son propre utilitarisme, son propre individualisme abstrait en montrant, dans la personne du neveu de Rameau, leur aboutissement problématique et paradoxal.

Il expose si exemplairement ce changement du monde et de soi par l'homme - la culture - et le renversement fatal de cette culture en son contraire, une nouvelle sorte de sauvagerie, que l'on a pu le considérer comme le témoin et l'auxiliaire le plus remarquable de la prise de conscience pré-révolutionnaire française, à la fin du XVIIIe siècle.

Plus que par la police ou par ses adversaires, Diderot a dû être effrayé par les conséquences extraordinaires, du point de vue thérique et du point de vue pratique, du constat terrible que le neveu de Rameau dressait sur un ton insolent et pittoresque. Ce n'est pas avec ses ennemis, dont les idées se trouvaient largement dépassées par ses analyses, qu'il livrait bataille. Mais c'est lui-même qui était le lieu d'un débat sans conclusion et d'un combat sans issue.

Car précisément, au débat qu'il ouvrait et au combat qu'il engageait - en grande part contre lui-même - la Révolution française ne saura apporter ni réponse, ni victoire.

$\mathrm{Si}$, dans l'Encyclopédie, nous avons affaire à l'homme abstrait, dans le Neveu de Rameau et dans Jacques le Fataliste, Diderot campe l'homme éminemment concret, situé exactement en son temps, en son pays, en sa ville, en sa classe de déclassé, en son milieu. Et les comparses sont aussi exactement typés : un monde irremplaçable.

Quand Jules Janin, à la fin du XIXe siecle, publiera le texte authentique du Neveu de Rameau, il ajoutera un sous-titre : Le Neveu de Rameau, suivi de l'analyse de la fin d'un monde. ${ }^{15}$

Les sarcasmes du Neveu de Rameau annoncent peut-être en effet la fin d'un monde, en tout cas ils révelent les contradictions et les déchirements internes de ce monde, le ver qui est dans le fruit.

Mais lorsque lon dit que le Neveu de Rameau annonce la fin d'un monde, on risque fort de se tromper de monde. Attention ! un monde en cache souvent un autre. En réalité, le Neveu de Rameau n'annonce pas la fin ultime de la féodalité, du pouvoir de l'aristocratie ou de la noblesse, de la monarchie. Il n'est question de rien de cela, dans la crise morale et sociale universelle que peint, à sa manière, le dialogue de

\footnotetext{
15 Paris: 1863.
} 
Diderot. Les aristocrates, les Grands, la Cour, le roi ne sont ni attaqués, ni critiqués dans le Neveu de Rameau. C'est comme s'ils n'existaient pas, comme si la Révolution française était déjã passée par là. Diderot ne veut pas leur donner le coup de pied de l'âne, même par anticipation. C'est à la puissance réelle et actuelle qu'il s'adresse : l'argent, le pouvoir économique nouveau, avant même qu'il ne se soit assuré le pouvoir politique. "L'or est tout"! s'exclame le neveu. "Quelle diable d'économie! Des hommes qui regorgent de tout, tandis que d'autres qui ont un estomac importun ccmme eux, une faim renaissante comme eux, n'ont pas de quoi mettre sous la dent"16 "Je ne vois partout que des esclaves et des tyrans"! Il veut être de ceux qui regorgent de tout, et il veut devenir un tyran.

A bien des égards, le neveu de Rameau préfigure ceux qui bientôt le surpasseront en infâmie et en cynisme, moins bons musiciens que lui, sans doute, mais plus heureux dans leurs affaires. Les brigands triompheront, ils survivront à toutes les révolutions et à toutes les restaurations, habiles à en tirer également profit.

Un jour, Chateaubriand verra passer devant lui les héritiers du neveu de Rameau, moins spirituels et moins sympathiques que lui dans sa misère, les parvenus, les triomphateurs, Talleyrand et Fouché, "le vice au bras du crime"...

Diderot avait pressenti leur irrésistible ascension.

Il a du être torturé, écartelé, épouvanté par cette vision de l'homme, de l'homme tel que nous le connaissons maintenant. Le monde du neveu, c'est le nôtre. Le neveu c'est notre voisin, notre maître, nous-même dans notre aliénation. Diderot est un critique et un moraliste de notre temps.

Il y a des commémorations qui tentent vainement de réveiller des morts. Diderot n'a pas besoin d'être réanimé, il nous introduit à la modernité et, quand nous le lisons, ce n'est pas nous qui le ressuscitons, c'est lui qui nous réveille encore.

${ }^{16}$ Oeuvres. Coll. Pléiade, 1951, p. 500. 